\title{
Rapid on-site evaluation (ROSE) for fine needle aspiration of thyroid: benefits, challenges and innovative solutions
}

\author{
Claire W. Michael ${ }^{1}$, Kaori Kameyama ${ }^{2}$, Wataru Kitagawa ${ }^{3}$, Nami Azar ${ }^{4}$ \\ ${ }^{1}$ Department of Pathology, University Hospitals Cleveland Medical Center/Case Western Reserve University, Cleveland, OH, USA; ${ }^{2}$ Department \\ of Pathology, School of Medicine, Keio University, Shinjuku-ku, Tokyo, Japan; ${ }^{3}$ Department of Surgery, Ito Hospital, Shibuya-ku, Tokyo, Japan; \\ ${ }^{4}$ Department of Radiology, University Hospitals Cleveland Medical Center/Case Western Reserve University, Cleveland, OH, USA \\ Contributions: (I) Conception and design: CW Michael; (II) Administrative support: None; (III) Provision of study materials or patients: All authors; \\ (IV) Collection and assembly of data: CW Michael; (V) Data analysis and interpretation: CW Michael; (VI) Manuscript writing: All authors; (VII) \\ Final approval of manuscript: All authors. \\ Correspondence to: Claire W. Michael, MD. Department of Pathology, MSPTH 5077, University Hospitals Cleveland Medical Center/Case Western \\ Reserve University, 11100 Euclid Ave Rm 212B, Cleveland, OH 44106, USA. Email: Claire.michael@uhhospitals.org.
}

\begin{abstract}
Rapid on-site evaluation/adequacy assessment (ROSE) is considered an essential component of thyroid fine needle aspiration (FNA) and many reported that it significantly decreases the nondiagnostic (ND) rate. The average reported ND rate without ROSE is about $20 \%$ and is improved by $12 \%$ when ROSE is implemented. However, the data also suggest that the improvement in ND rate after implementation of ROSE is directly related to the ND rate prior to ROSE and that it is mostly beneficial to aspirators with less experience. Several studies have also raised concerns regarding the impact of ROSE as it prolongs the procedure time, requires additional resources and increases the cost incurred by the additional fees. This resulted in a wide variation in the methodology applied to acquire the sample and implement ROSE across the globe with variation in the number of passes performed, stain utilized and the personnel reviewing the slides, e.g., cytotechnologists versus pathologist. This review summarized the literature reporting the impact of ROSE including its pros and cons, its accuracy and reproducibility, concordance between cytotechnologists and pathologists based on final diagnosis and highlights the different ways laboratories attempted to circumvent the challenges. In particular, the review highlights a unique approach practiced in Ito Hospital, Tokyo, Japan.
\end{abstract}

Keywords: Rapid on-site evaluation (ROSE); thyroid; fine needle aspiration (FNA); adequacy assessment

Submitted Apr 08, 2020. Accepted for publication Jul 24, 2020.

doi: $10.21037 /$ gs-2019-catp-23

View this article at: http://dx.doi.org/10.21037/gs-2019-catp-23

\section{Introduction}

Fine needle aspiration (FNA) biopsy is currently recommended as the primary method for the evaluation of a suspected thyroid nodule. While aspiration of palpable nodules by clinicians was a common practice in the past, ultrasound (US)-guided aspiration became the leading method in the last two decades especially since the guidelines recommend aspiration of all suspected thyroid nodules measuring $\geq 10 \mathrm{~mm}$ (1). The procedure is predominantly performed by radiologists although endocrinologists, surgeons and pathologists can also perform the procedure if properly trained and certified. As a result, the number of thyroid FNA exponentially increased and led to heightened demand on rapid onsite evaluation/adequacy assessment (ROSE), resulting in increased demand on the cytopathology laboratories' time, staff and resources. The following report reviews the current literature evaluating the impact of ROSE including both the benefits and challenges. The report also describes the various modalities laboratories developed to deliver the service. 


\section{How ROSE is performed}

While the technique of specimen preparation and how and who provides adequacy assessment may vary by institutions or geographic locations, the basic principal is the same. A staff is available onsite that handles the aspirated sample in real time. The most common practice across the globe is to deposit a drop of the sample on a slide and smear it by a second slide. A variety of quick stains are utilized depending on the preference of the individual laboratory such as Diff Quik (2-9), toluidine blue (10) and rapid Papanicolaou or hematoxylin and eosin $(4,11)$ stain. One slide is stained with a fast stain for immediate review while the other is fixed with alcohol. The needle is rinsed in a collecting media and later processed along with the fixed slide according to laboratory protocols.

\section{Impact of ROSE}

\section{Sample adequacy}

The reported rate of nondiagnostic (ND) FNA is as high as $20 \%(5,12-15)$. Several studies concluded that ROSE resulted in a significant decrease in the number of ND cases. In a Chinese study by Jiang et al. (10), the authors evaluated 1,103 FNA performed on suspicious thyroid nodules by two trained radiologists with at least 5 years of experience. Of those, 513 were performed with ROSE (WR) attended by a cytotechnologist and a pathologist who evaluated smears stained by Toluidine Blue. The remaining 590 cases were performed without Rose (WOR), in which the radiologists assessed the adequacy based on gross examination of the sample and the imaging findings. They concluded that while ROSE had no significant effect on the overall ND rate (NDR) (6.6\% WR vs. 9.5\% WOR), it resulted in significant reduction in NDR in certain nodules (sub-centimeter, mixed solid-cystic, macro-calcified and hyper vascular nodules). The NDR for other types of nodules was not significantly different. There was no significant difference in the incidence of complications. The mean number of passes was significantly reduced from $2.8 \pm 0.5$ in the WOR group to $1.7 \pm 0.6$ in the WR group $(\mathrm{P}<0.001)$.

In Brazil where nearly all procedures were performed by radiology residents, Pastorello et al. (4) evaluated a total of 4,649 FNA (3,469 WR and 1,180 WOR). The authors found that ROSE resulted in a significant difference with an adequacy rate of $93.4 \%$ for those $W R$ and $69.4 \%$ for those WOR $(\mathrm{P}<0.0001)$. The NDR WR was $6.6 \%$. While the operator routinely performed 3 passes when ROSE was not performed, the mean number of passes was reduced in the $W R$ group to $1.48 \pm 0.71$. No significant difference in the rate of malignancy among the two groups was noted.

In Colombia, Medina Chamorro et al. (2) evaluated 522 FNA and found that the NDR was $40 \%$ among the $197 / 522$ WOR and only $9.5 \%$ in the $325 / 522$ WR leading to a $30.5 \%$ decrease in the NDR.

Similarly in the USA, Zhu et al. (8) evaluated 833 cases and reported a significant reduction $(\mathrm{P}<0.001)$ in NDR from $31.8 \%(140 / 440)$ WOR to $5.9 \%(26 / 443)$ WR. Of interest, the authors found that follow up either by repeat FNA or resection of ND cases did not reveal any missed malignancy in the WR group while 7 carcinomas were found in the follow up of the WOR group. A meta-analysis by Witt et al. $(16,17)$ concluded that ROSE significantly improves specimen adequacy with an average adequacy of $92 \%$ in the WR group vs. $83 \%$ in the WOR group. However, the authors also suggested that based on gross inspection of the data, the improvement in adequacy due to ROSE may be related to the adequacy rate WOR. According to the metaanalysis by Schmidt et al. (17), studies with high adequacy rates WOR showed low improvement after ROSE was implemented and therefore had little opportunity for improvement.

On the other hand, others have questioned the value of ROSE. Ghofrani et al. (18) assessed a total of 981 USguided FNA and 521 thyroid FNA performed by palpation alone. The NDR for the entire group was $5.3 \% \mathrm{WR}$ and $10.5 \%$ WR. However the NDR for the US-guided group alone was $4.5 \%$ WR vs $7.1 \%$ WOR. The authors concluded that although ROSE significantly reduced the NDR among the entire group $(\mathrm{P}<0.01)$ as well as among FNA performed by palpation $(\mathrm{P}<0.025)$, the $2.6 \%$ reduction in NDR noted among US-guided cases was not significant.

\section{Time and cost}

In an effort to evaluate the cost effectiveness of ROSE, Eedes et al. (3) retrospectively reviewed 331 thyroid FNA. In their institution, such FNA could be performed by endocrinologist, surgeons or radiologist with and without ROSE. A thyroid clinic was also conducted twice a week to evaluate thyroid nodule referrals and staffed by an endocrinologist, a surgeon and a radiologist. They examined the NDR and calculated the time spent by cytology to provide ROSE. The NDR decreased from 14.9\% (32/215 WOR) to $9.5 \%$ (11/116 WR). Their estimated time spent for ROSE performed based on Diff Quik stain in the 
Thyroid Clinic was 35 and 57 minutes outside the Thyroid Clinic. The authors concluded that ROSE resulted in 23 additional adequate specimens in the WR group at a relatively high "cost" of 220 minutes of additional cytologist time per specimen and recommended the judicious use of ROSE. The authors also noted that ROSE was provided only within the hospital vicinity and that all the operators for these aspirates were highly experienced. Accordingly, if their results could be attributed to the operators, the bias would have been in favor of ROSE and US guidance. Selection bias could have also been a factor since ROSE tend to be requested for repeat FNA and those that are probably difficult to aspirate. Similarly, Tambouret et al. (19) reported that cytotechnologists spent about 70 minutes in performing ROSE and that radiologists with least experience had the highest benefit from ROSE. O'Malley et al. (11) raised a significant concern regarding the benefit of thyroid ROSE. The authors reviewed 50 retrospective and 21 prospective thyroid FNAs WR and compared them with 50 prospective FNA performed WOR. ROSE was performed by a cytopathologist based on examining on-site $\mathrm{H} \& \mathrm{E}$ stained slides. When they evaluated the 71 prospective FNA in which the total procedure time has been recorded, they found that ROSE prolonged the average procedure time from 12.5 to 44.4 minutes, without any significant decrease in the percentage of NDR $(24 \%$ vs. $20 \%$ ). About $50 \%$ of those WR and WOR were either limited or ND.

To assess the cost effectiveness of the additional cost and resources incurred by ROSE against the overall health care cost of managing a patient with a thyroid nodule, Nasuti et al. (5) reviewed 5,688 FNA performed in various organs WR. The authors compared the very low NDR $(0.98 \%)$ at their institution when ROSE is provided to the reported NDR of $20 \%$ for FNA WOR according to the literature. The authors concluded that despite the additional fees charged by cytology for ROSE, it was responsible for an estimated cost savings of $>\$ 400,000$ per year as a result of avoiding repeated aspirates (based on the assumption that all ND aspirates will be repeated). The authors also emphasized that their calculations may be underestimated because they did not take into account indirect cost to the patient such as additional time off work and longer hospital stays due to repeated biopsy.

\section{Number of passes}

When ROSE is not requested, 3-4 passes are routinely performed and grossly assessed by the operator. However, when ROSE is also performed, the number of passes vary since the operator continues to perform new passes until an adequate verdict is issued. Several studies attempted to assess the optimal number of passes. Eedes et al. (3) reported that $66 \%$ of their cases were deemed adequate with 2 passes and $98 \%$ with 3 or fewer passes and that additional passes were not useful. Additional studies have also demonstrated that the significant increase in diagnostic yield was after the second pass and that additional passes gave little further improvement (20,21). Zhu and Michael (8) correlated the number of passes with adequacy in their cases. They found that the number of ND cases decreased from $25 \%$ to $11.0 \%(\mathrm{P}<0.001)$ in the $\mathrm{WR}$ procedures when 4 passes were performed. Their data demonstrated a continuous reduction of ND cases with the increase in number of FNA passes up to 6 passes. Additional passes did not significantly improve adequacy.

\section{Concordance between ROSE and final diagnosis}

In the report by Eedes et al. (3), the authors noted that criteria for adequacy varied somewhat among their cytologists. Generally, they considered a specimen adequate when several groups of at least a dozen follicular cells were present and that they required less cellularity when abundant colloid was identified. Of the cases deemed adequate By ROSE in their study, 20\% had suboptimal cellularity upon final review. The authors explained that there seems to be a tendency by pathologists to consider borderline samples as adequate assuming that additional material will be seen on the TP, however such expectation may not always materialize. Meanwhile, Jing et al. (7) retrospectively evaluated their concordance rate after their institution self- implemented a standardized requirement of at least 6 clusters of follicular cells, 10 cells each on the examined Diff-Quik slides. There was total agreement on specimen adequacy in $93 \%$ of cases between adequacy by ROSE and final assessment. Adequacy was converted in $70 / 178$ samples from ND to diagnostic while only $2 / 853$ specimens were reversed to ND on final diagnosis. Their cytohistologic concordance rate was $93.9 \%$ for nonneoplastic nodules and $82.3 \%$ for neoplastic ones.

To assess concordance rate for adequacy provided by cytotechnologists on-site versus pathologists, Olson (6) retrospectively reviewed 2,261 cases, $64.7 \%(n=1,462)$ attended by cytotechnologists and $35.3 \%(\mathrm{n}=799)$ attended by cytopathologists. The authors found no difference in the 
adequacy downgrade rate for cytotechnologists compared with that for cytopathologists ( $4.1 \%$ vs. $5 \%$ ).

\section{Discussion}

FNA is an important triage tool for assessing thyroid nodules and evaluate for malignancy. The majority of the literature favors the value of utilizing ROSE during the procedure and confirms that it results in lower NDR. The Bethesda Reporting System for Thyroid Aspirates recommends the use of ROSE when possible and particularly in repeat aspirates $(22,23)$. However, several studies as described above, have either doubted the value of ROSE or raised concerns primarily regarding the related cost, prolonged procedure time and the burden imposed on the cytology laboratories to provide this service.

The variation among studies may be a result of multiple factors. For example, the nodule size requirement set by different institutions may affect the NDR since the smaller the size, the higher the expected NDR (24). The stain protocol used by pathology can impact the time required for ROSE. Some studies performed ROSE on fixed and $H \& E$ stained slides (11). This technique requires at least 2 minutes and necessitate the use of mounting media and coverslip. Others described using air dried Diff-Quik stained $(3,8)$ or Toluidine Blue stained slides $(10)$, both are done within 30-40 seconds and require no mounting media. The latter, would save at least one minute per pass. Also, the reported operator experience varied among studies ranging from residents to well experienced operators. In fact, according to the meta-analysis by Schmidt et al. (17), while on average, ROSE improved the adequacy rate by $12 \%$, there was considerable variability across studies. Based on the data, $65 \%$ of the variability in the adequacy rate WR was found to occur because of differences in the adequacy rate WOR. Based, on these studies, the value of ROSE seems to be highest when the operators are less experienced.

Whether ROSE is still worth the additional cost or not is still controversial. There is no doubt that the exponential increase in the number of thyroid FNA in the last two decades have put tremendous burden on the cytology laboratories. First, not all laboratories have the resources to offer ROSE which requires mobilizing the cytotechnologist or trainee to the FNA site; time spent away from doing other duties in pathology; and in some countries the mandate for a cytopathologist presence as well. Second, the reimbursement fees vary tremendously depending on the third party payers, and regulations imposed by such payers. For example, some healthcare payers put a cap on procedure fees regardless of whether ROSE or other ancillary tests were performed or not. In the USA, where many third party payers still reimburse for ROSE, the Medicare and Medicaid impose a ruling that for any charged fee, a pathologist has to be present and personally reviews the key portion of the procedure. As a result, even when cytotechnologist or trainee are available and quite capable to perform ROSE, a pathologist is still required to personally review the slides and provides the assessment of adequacy (key portion).

Some may argue that the value of ROSE outweighs its additional cost and that it results in decrease in the overall healthcare cost (5). This conclusion is based on the assumption that ND cases will require repeat aspiration which will inflict additional cost on both the health care system and the patient. Others caution against the routine use of ROSE and recommend judicious use (3). Layfield et al. (25) analyzed the compensation for ROSE which varied by healthcare providers and concluded that ROSE is insufficiently compensated by current Medicare.

\section{ROSE: to do it or not to do it?}

The decision whether to offer ROSE or not lies in each institution individually depending on its own resources, patient population and fee coverage. As a result, institutions have responded to the need for ROSE in various ways. Some institutions situated pathologists attending ROSE to perform their routine duties in a location adjacent to the ultrasonography/procedure rooms thus minimizing the time spent by the cytopathologist (4). Many institutions adopted selective use of ROSE only for suspicious nodules or for repeat FNA. Who prepares the smears also vary by institution, some will still send their cytotechnologists or trainee while others elected to train the radiology staff to prepare and stain the smears. Others instituted telecytology systems and reported great results $(9,26)$, where cytotechnologists prepare, stain and screen the slides then connect with the cytopathologist's working station using a secure server to transmit in time the reviewed slides and project the concerning areas. This procedure saves the cytopathologist time, yet still requires cytotechnologist attendance. However, such success relies heavily on well trained and capable cytotechnologists since they lead the pathologist through the transmitted images.

The first author had the pleasure of visiting Ito Hospital in Tokyo Japan where she observed their different approach 


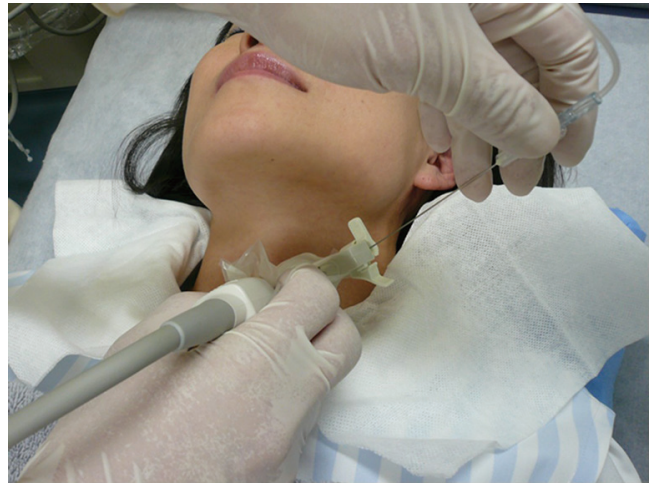

Figure 1 Patient lies in a supine position and asked to look up. The nodule and safest path for FNA is located using the US probe. FNA, fine needle aspiration; US, ultrasound.

to thyroid FNA and ROSE. Ito Hospital deals exclusively with the evaluation and management of thyroid nodules. Consequently they perform a large number of US-guided FNA on a daily basis but a cytopathologist attends the hospital three times a week only to review the cases. While in the USA and many other countries including Japan, the free hand technique is the preferred method for USguided aspirations, Ito Hospital utilizes a slightly different technique as described below (27).

While US-guided FNA technique is beyond the scope of this review, a short description to highlight the different methods is warranted. In general, the patient lies in a supine position and is asked to look up. Once the thyroid nodule is identified using US and the vascularity of the nodule is assessed by Doppler, the safest access to avoid large vessels is mapped. The site is prepped in the regular, sterile fashion and the appropriate sterile coverage is applied to maintain sterility; $1 \%$ lidocaine is used as a local anesthetic (some providers use $4 \%$ lidocaine topical anesthetic cream or perform the procedure without any local anesthetics). Then a sterile. Probe cover is applied to maintain site sterility. With US-guidance, using a freehand technique, the nodule is accessed with a 22-27-gauge needle (most commonly 25 -gauge). After removing the needle styloid, the needle is moved from one side to another of the nodule without applying any suction (capillary action). Needle motion is continuously monitored during sample acquisition. A minimum of 5 cycles of to-and-fro is recommended (27). In Ito Hospital, the patient is prepped in the same way but no local anesthesia is used. The US probe used has a mounted needle guidance bracket (Figure 1). A 22 -gauge needle, $120 \mathrm{~mm}$ long (TOP Corporation, Tokyo, Japan) is attached

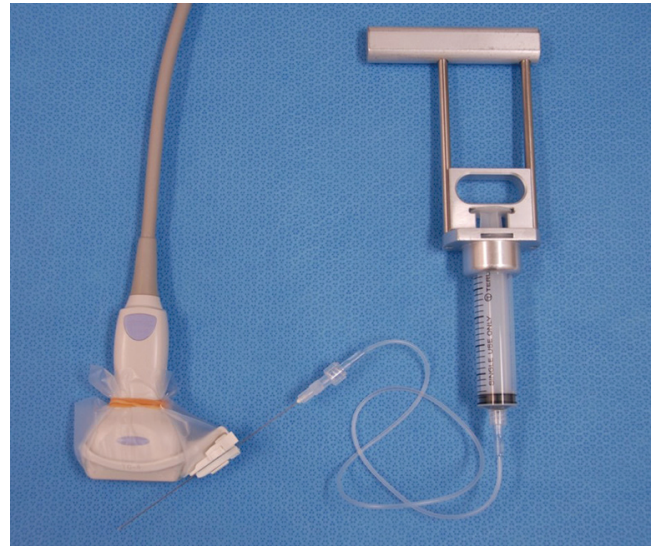

Figure 2 The US probe used has a mounted needle guidance bracket. A 22-gauge needle, $120 \mathrm{~mm}$ long (TOP Corporation, Tokyo, Japan) is attached to a $20 \mathrm{~mL}$ syringe with an extension tube. US, ultrasound.

to a $20 \mathrm{~mL}$ syringe with an extension tube (Figure 2). After confirming the needle tip is in the target nodule under USguidance by the operator, negative pressure is generated by the assisting nurse using a pestle type syringe holder. The operator rotates the needle between their thumb and index finger in a fast motion to collect the sample. Then the negative pressure is released and the needle is removed and handed to a medical technologist for processing and adequacy assessment. Only one pass is performed unless the sample is deemed inadequate. It is worth noting that while the utility of the needle guidance mounted on the probe cover with the plastic attachment is slightly more expensive, it does provide easier and faster access to the nodule. However, the free hand technique provides more flexibility with the approach (not locked to specific angel set by the guide) and consequently the operator can use a perpendicular, parallel or both approaches while obtaining the sample. Rotating the needle during aspiration improves sample adequacy.

In Ito Hospital, once the needle is handed to the medical technologist, a small portion is dropped on a slide and smeared in the conventional method with one slide air dried for Diff-Quik stain and the other slide fixed in ethanol for Papanicolaou stain (both stains performed later in the laboratory). The remaining sample is processed on site using a filter apparatus (Senshin Medical Inc.). In short a filter is installed within the apparatus and a cylinder is installed above the filter. The cylinder is filled with lactate Ringer solution-Saviosol ${ }^{\mathrm{TM}}$ adjusted osmotic pressure with 

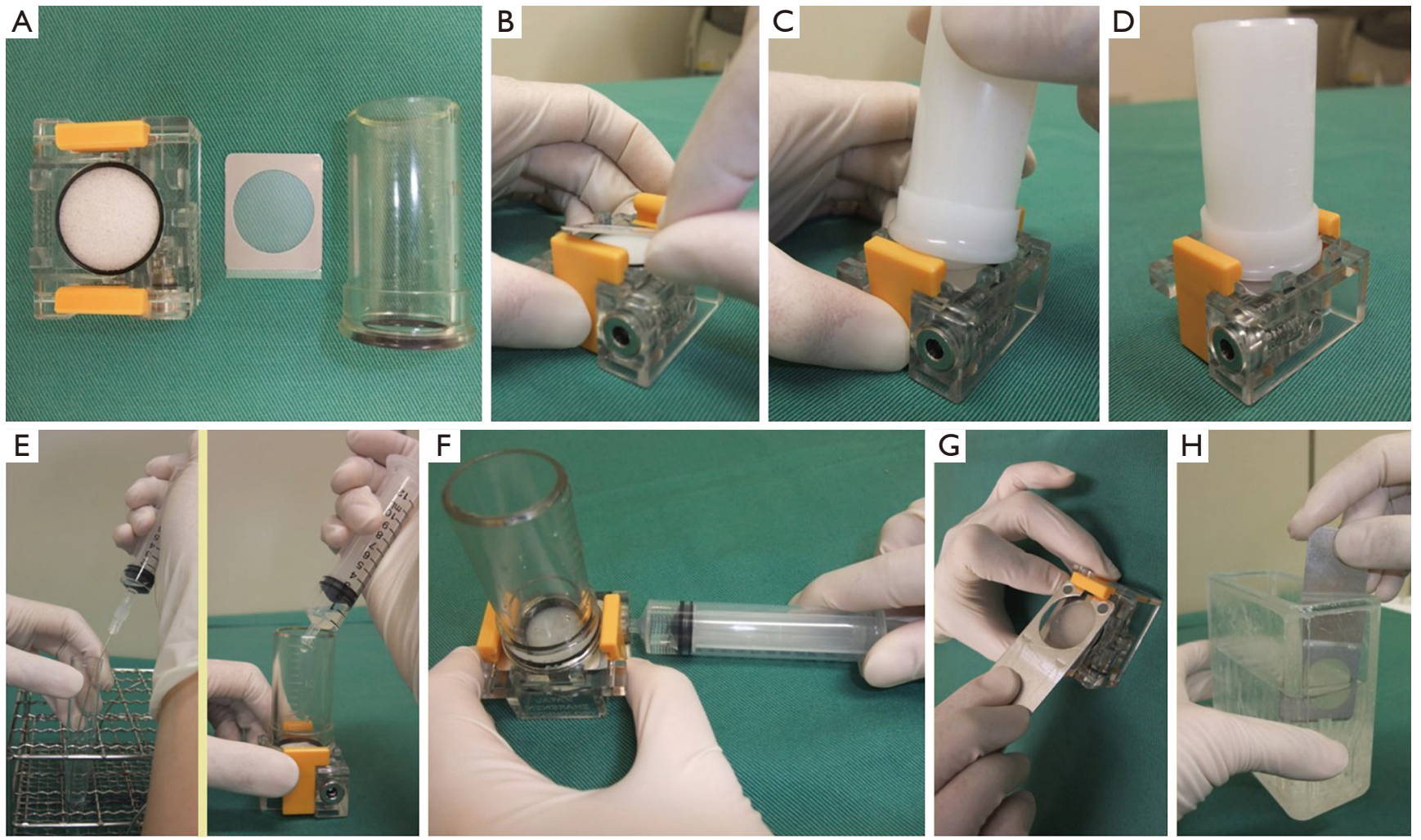

Figure 3 Use of the filter apparatus. (A) Components consist of the main apparatus, a filter and a cylinder; (B) the filter is installed; (C) the cylinder is installed above the filter; (D) apparatus ready to use; (E) cylinder is filled with Ringer solution and specimen is rinsed in the solution; (F) negative pressure is applied to the cylinder; $(\mathrm{G})$ the filter is grossly examined and $(\mathrm{H})$ filter is put in ethanol fixative.

low molecular weight dextran 40 (Otsuka pharmaceutical Co. Ltd, Tokyo, Japan). The needle is rinsed in the Ringer solution and a negative pressure is applied to pull the solution containing the needle rinse through the filter. The filter is removed and grossly examined for adequacy and then fixed in ethanol for Papanicolaou stain (Figure 3). Their reported NDR for a total of 14,668 FNA from 11,830 patients was $1,066(7.3 \%)$. However, when cyst fluid only cases $(732 / 1,066$ or $5 \%)$ that are diagnosed as benign per Japan Reporting System are excluded, the NDR is only $2.3 \%$ (334 cases) (27).

ROSE plays a much larger role that goes beyond providing adequacy assessment of the sample or a preliminary diagnosis. The immediate feedback provided by pathology can impact the operator in several ways. First, it assists the operator in deciding the best needle path and whether it needs to be differently oriented. Second, it provides on time feedback that enhances the skill development of the operator particularly the less experienced and/or trainee. Third, the presence of pathology results in direct correlation of the ultrasonographic appearance of the mass with the correlating morphology thus facilitating further decisions, e.g., acquiring a concurrent biopsy, additional passes, etc.

While therapeutic decisions are not made based on the preliminary diagnosis, it is still essential to triage the sample for further work-up and ancillary tests. For example, a suspicion for medullary carcinoma will trigger the collection of additional passes beyond simple adequacy to prepare a cell block for immunochemistry. Similarly, when lymphoma is suspected the sample can be triaged for flow cytometry. As a result, the specimen is appropriately handled and repeat procedures to collect for the ancillary tests can be avoided. With the integration of molecular testing of thyroid FNA for indeterminate diagnoses, ROSE is becoming essential to achieve an adequate sample for both diagnosis and testing.

In conclusion, whether the sample is triaged in the first FNA utilizing ROSE or differed to repeat procedure selectively utilizing ROSE or intraoperative consultation, i.e., frozen section is decided by the individual institutions 
as they weigh the value of ROSE against the incurred expenses and available resources.

\section{Acknowledgments}

We would like to acknowledge Professor Kennichi Kakudo for his contribution in this work as he introduced the authors, translated the Japanese references, and insured accuracy of the information.

Funding: None.

\section{Footnote}

Provenance and Peer Review: This article was commissioned by the Guest Editor (Kennichi Kakudo) for the series "Asian and Western Practice in Thyroid Pathology: Similarities and Differences" published in Gland Surgery. The article was sent for external peer review organized by the Guest Editor and the editorial office.

Conflicts of Interest: All authors have completed the ICMJE uniform disclosure form (available at http://dx.doi. org/10.21037/gs-2019-catp-23). The series "Asian and Western Practice in Thyroid Pathology: Similarities and Differences" was commissioned by the editorial office without any funding or sponsorship. The authors have no other conflicts of interest to declare.

Ethical Statement: The authors are accountable for all aspects of the work in ensuring the questions related to the accuracy or integrity of any part of the work are appropriately investigated and resolved.

Open Access Statement: This is an Open Access article distributed in accordance with the Creative Commons Attribution-NonCommercial-NoDerivs 4.0 International License (CC BY-NC-ND 4.0), which permits the noncommercial replication and distribution of the article with the strict proviso that no changes or edits are made and the original work is properly cited (including links to both the formal publication through the relevant DOI and the license). See: https://creativecommons.org/licenses/by-nc-nd/4.0/.

\section{References}

1. Haugen BR, Alexander EK, Bible KC, et al. 2015 American Thyroid Association Management Guidelines for Adult Patients with Thyroid Nodules and Differentiated Thyroid
Cancer: The American Thyroid Association Guidelines

Task Force on Thyroid Nodules and Differentiated Thyroid Cancer. Thyroid 2016;26:1-133.

2. Medina Chamorro FM, Calle JA, Stein JE, et al. Experience of the Implementation of Rapid On-Site Evaluation in Ultrasound-Guided Fine-Needle Aspiration Biopsy of Thyroid Nodules. Curr Probl Diagn Radiol 2018;47:220-4.

3. Eedes CR, Wang HH. Cost-effectiveness of immediate specimen adequacy assessment of thyroid fine-needle aspirations. Am J Clin Pathol 2004;121:64-9.

4. Pastorello RG, Destefani C, Pinto PH, et al. The impact of rapid on-site evaluation on thyroid fine-needle aspiration biopsy: A 2-year cancer center institutional experience. Cancer Cytopathol 2018;126:846-52.

5. Nasuti JF, Gupta PK, Baloch ZW. Diagnostic value and cost-effectiveness of on-site evaluation of fine-needle aspiration specimens: review of 5,688 cases. Diagn Cytopathol 2002;27:1-4.

6. Olson MT, Tatsas AD, Ali SZ. Cytotechnologistattended on-site adequacy evaluation of thyroid fineneedle aspiration: comparison with cytopathologists and correlation with the final interpretation. Am J Clin Pathol 2012;138:90-5.

7. Jing X, Wey E, Michael CW. Retrospective evaluation of instituted standard adequacy criteria for on-site adequacy assessment of thyroid fine-needle aspiration. Diagn Cytopathol 2011;39:391-4.

8. Zhu W, Michael CW. How important is on-site adequacy assessment for thyroid FNA? An evaluation of 883 cases. Diagn Cytopathol 2007;35:183-6.

9. Lin DM, Tracht J, Rosenblum F, et al. Rapid On-Site Evaluation With Telecytology Significantly Reduced Unsatisfactory Rates of Thyroid Fine-Needle Aspiration. Am J Clin Pathol 2020;153:342-5.

10. Jiang D, Zang Y, Jiang D, et al. Value of rapid on-site evaluation for ultrasound-guided thyroid fine needle aspiration. J Int Med Res 2019;47:626-34.

11. O'Malley ME, Weir MM, Hahn PF, et al. US-guided fineneedle aspiration biopsy of thyroid nodules: adequacy of cytologic material and procedure time with and without immediate cytologic analysis. Radiology 2002;222:383-7.

12. Bohacek L, Milas M, Mitchell J, et al. Diagnostic accuracy of surgeon-performed ultrasound-guided fineneedle aspiration of thyroid nodules. Ann Surg Oncol 2012;19:45-51.

13. Haider AS, Rakha EA, Dunkley C, et al. The impact of using defined criteria for adequacy of fine needle aspiration 
cytology of the thyroid in routine practice. Diagn Cytopathol 2011;39:81-6.

14. Jo VY, Stelow EB, Dustin SM, et al. Malignancy risk for fine-needle aspiration of thyroid lesions according to the Bethesda System for Reporting Thyroid Cytopathology. Am J Clin Pathol 2010;134:450-6.

15. Theoharis CG, Schofield KM, Hammers L, et al. The Bethesda thyroid fine-needle aspiration classification system: year 1 at an academic institution. Thyroid 2009; 19:1215-23.

16. Witt BL, Schmidt RL. Rapid onsite evaluation improves the adequacy of fine-needle aspiration for thyroid lesions: a systematic review and meta-analysis. Thyroid 2013;23:428-35.

17. Schmidt RL, Witt BL, Matynia AP, et al. Rapid on-site evaluation increases endoscopic ultrasound-guided fineneedle aspiration adequacy for pancreatic lesions. Dig Dis Sci 2013;58:872-82.

18. Ghofrani M, Beckman D, Rimm DL. The value of onsite adequacy assessment of thyroid fine-needle aspirations is a function of operator experience. Cancer 2006;108:110-3.

19. Tambouret R, Szyfelbein WM, Pitman MB. Ultrasoundguided fine-needle aspiration biopsy of the thyroid. Cancer 1999;87:299-305.

Cite this article as: Michael CW, Kameyama K, Kitagawa W, Azar N. Rapid on-site evaluation (ROSE) for fine needle aspiration of thyroid: benefits, challenges and innovative solutions. Gland Surg 2020;9(5):1708-1715. doi: 10.21037/gs2019-catp-23
20. Civardi G, Fornari F, Cavanna L, et al. Value of rapid staining and assessment of ultrasound-guided fine needle aspiration biopsies. Acta Cytol 1988;32:552-4.

21. Padhani AR, Scott WW Jr, Cheema M, et al. The value of immediate cytologic evaluation for needle aspiration lung biopsy. Invest Radiol 1997;32:453-8.

22. Cibas ES, Ali SZ. The 2017 Bethesda System for Reporting Thyroid Cytopathology. Thyroid 2017;27:1341-6.

23. Ali SZ, Cibas ES. The Bethesda System for Reporting Thyroid Cytopathology. New York: Springer; 2010.

24. Sabel MS, Haque D, Velasco JM, et al. Use of ultrasoundguided fine needle aspiration biopsy in the management of thyroid disease. Am Surg 1998;64:738-41; discussion 741-2.

25. Layfield LJ, Bentz JS, Gopez EV. Immediate on-site interpretation of fine-needle aspiration smears: a cost and compensation analysis. Cancer 2001;93:319-22.

26. Canberk S, Behzatoglu K, Caliskan CK, et al. The Role of Telecytology in the Primary Diagnosis of Thyroid FineNeedle Aspiration Specimens. Acta Cytol 2020;64:323-31.

27. Gill AS, Amdur R, Joshi AS. Importance of FNA

Technique for Decreasing Non-diagnostic Rates in Thyroid Nodules. Head Neck Pathol 2018;12:160-5. 